\author{
Military Technical College \\ Kobry Elkobbah, \\ Cairo, Egypt \\ May 16-18,2006
}

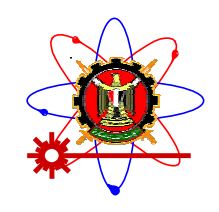

\title{
Analysis and Optimization of the Laser Speckles Produced by Different Light Sources to Design a New Optical Sensor
}

\author{
Hatem El-Ghandoor ${ }^{\mathrm{a}}$, Ashraf F. El-Sherif ${ }^{\mathrm{b}}$ and Mohamed Darwish ${ }^{\mathrm{c}}$ \\ ${ }^{a}$ Faculty of Sciences, Ain Shams University, Cairo, Egypt \\ ${ }^{b, c}$ Egyptian Armed Forces
}

\section{Abstract}

The basic elements of the optical computer mouse (OCM) are; a light emitting diode (LED), image acquisition system (IAS) which acquires images via the lens and a digital signal processor (DSP) to implement the algorithm to determine direction and distance of motion. Here, we describe the light speckles produced from different colour LEDs and laser sources to design and implement a new optical computer mouse. The speckle pattern will be used also to determine the responsivity and velocity of the device relative to the surface it slides on it. The most important and critical property of speckles is their average diameter, which is independent of the type of the surface being illuminated by coherent $(\mathrm{He}-\mathrm{Ne}$ laser and diode laser) or partially coherent light (LEDs). The average diameter of a speckle pattern is function of the diameter of the illuminated area of the pad surface, the distance between the pad surface and the detector, and the wavelength of the used light.

In this work, we replaced the OCM light source by a small powerful coloured LEDs and laser sources and studying the resulting coloured speckle patterns to investigate the effect of different wavelengths and the distance between the pad surface and the detector on the speckle diameters which affects the response of the OCM.

Keywords: Laser Speckle Photography, LEDs, Optical computer mouse, Digital image processing, Threshold detector.

\section{Introduction}

When a coherent light rays is incident on an optically rough object it will scatter randomly through all directions. Interference of these scattered rays leads to the formation of a bright spots (constructive interference), and dark spots (destructive interference), these are called Laser Speckles as shown in Fig.1. [1-4]. The rough objects or diffuse objects are usually of two types: diffuse reflecting objects and transparent diffuse objects, the rough surfaces of stones, cement, unpolished metal, wood, and so on are 'Diffuse reflecting objects'. Ground glass is a well-known example of a 'Transparent diffuse object'. It is difficult to determine a lower limit under which an object is considered well polished, because it is not only the thickness variations that count, but their spatial distribution: in other terms, the slopes of the profile of the diffuse object. Double exposures one before and one after displacement of the rough surface, when recorded in the same emulsion leads to the formation of speckle pairs. The two recorded 
speckle patterns were processed and then illuminated by a coherent light beam. For lateral in plane displacement by the double exposure speckle photography technique, the speckle pairs (shifted one and original one) lead to Young's cosine interference fringes.

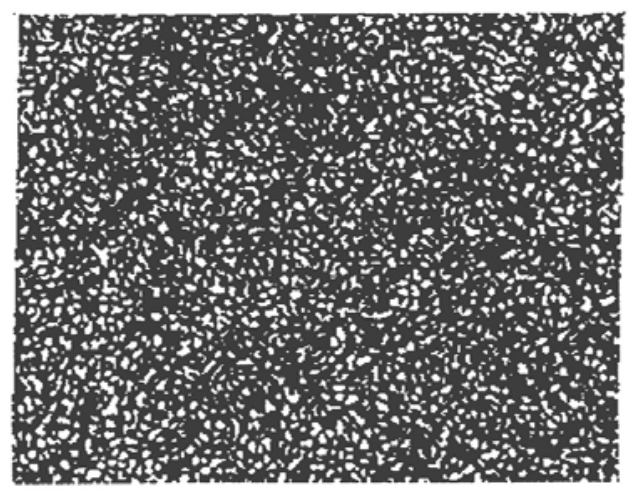

Fig. 1. A speckle pattern

Consider one type of two any diffuse objects, for instance, a transparent diffuse object such as a ground glass Fig .2. It is illuminated by an extended light source; a lens $(O)$ with focal length $(I)$ will be used to form an image of this object at the focal plane $\left(\pi^{\prime}\right)$. Each point of the object has a diffraction pattern whose dimensions depend only on the used image-forming lens. All points of the object $(G)$ scatter coherent light waves that are capable of interfering to produce laser speckles.

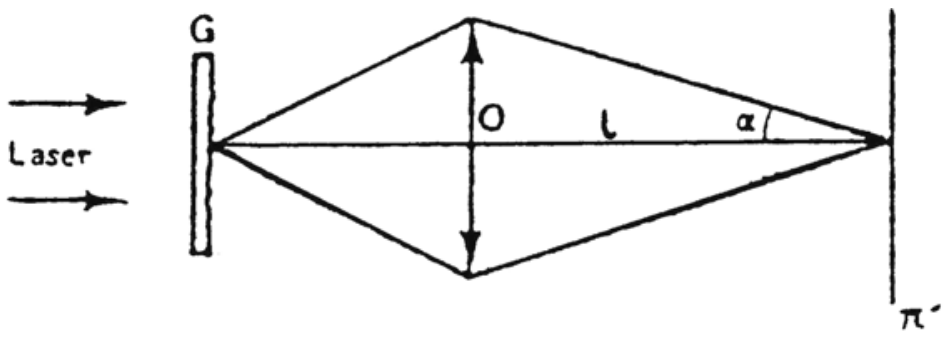

Fig. 2. Transparent diffuse object

\subsection{Speckle Photography Technique}

The simplest technique to set up and operate in speckle metrology is Laser speckle photography (LSP). LSP is an optical technique that is used for measuring many variables such as displacement, velocity, the relative velocity between two objects [5] and refractive index profile. The general procedure consists of exposing high resolution photographic plate to two displaced speckle patterns, one before and one after a change is introduced to the diffuse object. This is called double-exposure speckle photography. The film that results from a speckle photography process is called specklegram.

When a fine laser beam is directed onto a certain point on the specklegram, this point includes pairs of the speckles separated by $\Delta$ which may be considered as two point sources displaced by a distance $\Delta$, then Young's fringes shown in Fig.3. of angular spacing $\lambda / \Delta$ are observed on the screen at 
distance $l$ from the specklegram. The interfringe spacing $s$ is given by: $s=\lambda l / \Delta$

(1)

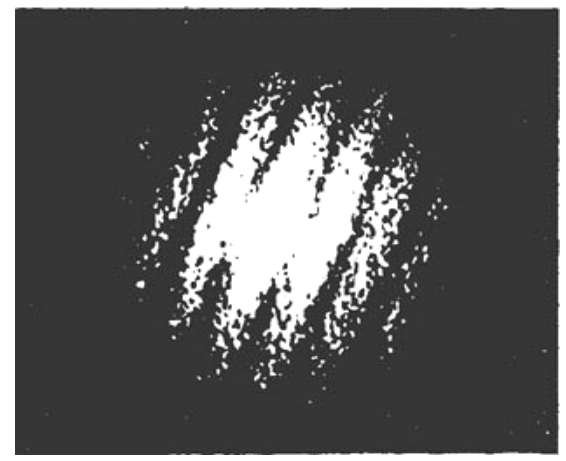

Fig. 3. Interference fringes from double exposure speckle photography technique

\section{Theoretical Explanations:}

The most important and critical property of speckles is that their average diameter which is almost independent of the type of the surface being illuminated by coherent or partially coherent light. The average diameter of a speckle pattern is function of the diameter " $d$ " of the illuminated area of the surface, the distance $L$ between the surface and the detector, and the wavelength of the used light is $\lambda$. The average speckle diameter $\delta$ is given by [6]:

$$
\delta=\frac{1.2 \lambda L}{d}
$$

When the used light is a laser and is allowed to be incident normally on the rough surface where the scattering centres are distributed randomly we can get an isotropic speckle pattern (ISP). The term isotropic means that the probability of obtaining speckles with a certain diameter are the same through all directions. Also it is very important to study the behaviour of the speckle pattern when the scattering surface is moving along certain directions. This movement will destroy the old speckle pattern while a new speckle pattern will be generated. This is due to the fact that speckle pattern is a result of constructive and destructive interference of the scattered light.

The probability density function of the intensity distribution of speckle formed follows the exponential form [6]:

$$
P(I)=\left(I / I_{o}\right) e^{\left(-I / I_{o}\right)}
$$

Where $P(I)$ is the probability that a particular speckle has an intensity between $I$ and $\left(I+I_{o}\right)$. It is clear that the most probable brightness of speckles is zero, i.e. there are more completely dark speckles than those of any other brightness.

The complex amplitude of the incident light from the optical mouse in the form of a spherical wave starting from $x=0, y=0$ and $z=0$ as given by:

$$
U(r)=\frac{a}{r} e^{i k r}=\frac{a}{r} e^{\left[i \frac{2 \pi}{\lambda}\left(x^{2}+y^{2}+z^{2}\right)^{1 / 2}\right]}
$$


At a distance $L$ from the mouse in a plane normal to the $z$-axis this amplitude will be:

$$
U(x, y, L)=\frac{a}{r} e^{i k r}=\frac{a}{r} e^{\left[i \frac{2 \pi}{\lambda} \cdot\left(x^{2}+y^{2}+L^{2}\right)^{1 / 2}\right]}
$$

For a point closer to the $z$-axis $L$ is very large with respect to $\mathrm{x}$ or $\mathrm{y}$ : then the following approximation can be made:

$$
\left(x^{2}+y^{2}+L^{2}\right)^{1 / 2}=L\left\{1+\frac{1}{2}\left[\frac{x^{2}+y^{2}}{L^{2}}\right]\right\}
$$

Then:

$$
U(x, y, L)=\frac{a}{r} e^{\left[i \frac{2 \pi}{\lambda} L\right]} e^{\left[\frac{2 \pi i}{2 L \lambda}\left(x^{2}+y^{2}\right)\right]}
$$

As $\mathrm{x}, \mathrm{y}<<L$ then $r \approx L$ and we can write:

$$
U(x, y, L)=\frac{a}{L} e^{\left[i \frac{2 \pi}{\lambda} L\right]} e^{\left[\frac{\pi i}{L \lambda}\left(x^{2}+y^{2}\right)\right]}
$$

An optical mouse is rested on a small area on the transparency pad, which is centred on position $R(x, y)$ sends at any point on the detector like $M(X, Y)$ the speckle amplitude $d A 1$, where:

$$
d A_{1}=d\left[A_{1}(X, Y)\right]=a(X, y) e^{\left[\frac{i \pi\left[(X-x)^{2}+(Y-y)^{2}\right]}{\lambda L}\right]} d x d y
$$

Where $\mathrm{a}(x, y)$ is the transparency of the pad and $L$ is the distance between the detector of the optical mouse and the pad surface. Then at $R(x, y)$ the total transmitted amplitude to $M(X, Y)$ is

$$
\begin{gathered}
A_{1}(X, Y)=\iint_{\text {area }} d A_{1} \\
A_{1}(X, Y)=e^{\left[\frac{i \pi\left(X^{2}+Y^{2}\right)}{\lambda L}\right]} \iint_{\text {area }} a(x, y) e^{\left[\frac{i \pi\left(x^{2}+y^{2}\right)}{\lambda L}\right]} e^{\left[\frac{-2 i \pi}{\lambda L}(X x+Y y)\right]} d x d y
\end{gathered}
$$

The received intensity will be:

$$
\begin{aligned}
& I_{1}(X, Y)=\left|\mathrm{A}_{1}(\mathrm{X}, \mathrm{Y})\right|^{2} \\
& =\left|e^{\left[\frac{i \pi\left(X^{2}+Y^{2}\right)}{\lambda L}\right]} \iint_{\text {area }} a(x, y) e^{\left[\frac{i \pi\left(x^{2}+y^{2}\right)}{\lambda L}\right]} e^{\left[\frac{-2 i \pi}{\lambda L}(X x+Y y)\right]} d x d y\right|^{2}
\end{aligned}
$$

After a small shift $€$ of the optical mouse on the pad surface the distance $L$ will be replaced by $\left(L^{2}+\varepsilon^{2}\right)^{\frac{1}{2}} \approx L\left(1+\frac{\varepsilon^{2}}{2 L^{2}}\right)$, this means that the received intensity at the new position of the mouse is given by: 
$I_{1}(X, Y)=\left|e^{\left[\frac{i \pi\left(X^{2}+Y^{2}\right)}{\lambda L\left(1+\varepsilon^{2} / 2 L^{2}\right)}\right]} \iint_{\text {area }} a(x, y) e^{\left[\frac{i \pi\left(x^{2}+y^{2}\right)}{\lambda L\left(1+\varepsilon^{2} / 2 L^{2}\right)}\right]} e^{\left[\frac{-2 i \pi}{\lambda L\left(1+\varepsilon^{2} / 2 L^{2}\right)}(X x+Y y)\right]} d x d y\right|^{2}$

Consider" n" to be the number of scattering centres within the illuminated area of the surface. When the surface moves with velocity $(\mathrm{V})$ in time interval " $\Delta \mathrm{t}$ ", it moves a distance:

$$
\Delta \mathrm{x}=\mathrm{v} \Delta \mathrm{t}
$$

Now some of the scattering speckles from the surface will be lost, let its number to be $(\Delta \mathrm{n})$,, but also other new speckles will be generated, let its number to be $(\Delta n)_{+}$. This is due to the lack of correlation between the speckle patterns formed by different scattering centres. Nevertheless, if $\Delta x<<$ and hence $(\Delta \mathrm{n})_{-} \ll \mathrm{n}$ and $(\Delta \mathrm{n})_{+}<<\mathrm{n}$, it can be expected that the same speckle pattern occurs but just shifted by a distance $\Delta \mathrm{x}$, with each individual speckle retaining its position, shape and diameter. This means that

in order to obtain a new optical computer mouse suggested in this paper to work correctly, the following conditions should be verified:-

$$
\delta<\Delta \mathrm{x}<<\quad \text { and } \quad \delta \leq b
$$

Where $b$ is the average size of the dark speckle or the separation between two bright speckle spots. This condition is achieved by considering all the speckle spots to acquire a circular shape with average diameter $\delta$ of the bright speckle.

In this work we can control the sizes of the dark speckle $(b)$ and the bright speckle $(\delta)$ by changing either the distance between the optical mouse detector and the pad surface $(L)$ or the wavelengths of the LEDs or laser sources $(\lambda)$.

\subsection{The Operation of optical computer mouse:}

The optical mouse illuminates an area of work surface with a LED, to reveal a microscopic speckle pattern (highlights and shadows). Optical mouse is a complete imaging system as shown in Fig.4. Assuming that the surface which scatters light is moving with respect to the sensor with velocity $(v)$. The optical sensor provides a non mechanical tracking engine for implementing our computer mouse. On this sensor the IC chip, images are captured, digitized, and then digitally processed, where it contains five photodiodes labelled $U, D, L, R$ and $C$ are positioned at the vertices and the centre of a square. They are used to sense over the speckle pattern and to convert the speckle grain into electric signal. The sensing area of photodiode is "a" and it must be of the same range of the average diameter of the speckle grains i.e.: $\mathrm{a} \approx \delta$.

Each photodiode (PD) is attached to its own threshold detector (TD). All TD have identical threshold values. The PDs and the TDs make up the optical sensor. The LED and the sensor should be worked up to form a solid construction. 


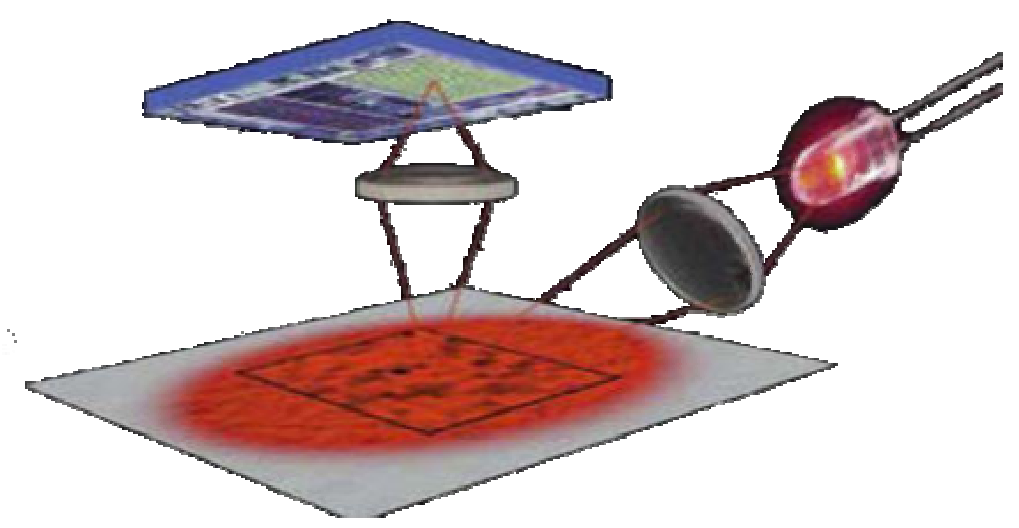

Fig. 4. Optical computer mouse (OCM) as a complete imaging system.

The output signal from the TD has two levels: level $\mathrm{H}$ (high) that refers to the case when the attached $P D$ is illuminated above the threshold value and level $L$ (low) indicates that the PD is illuminated under the threshold value. Each TD output is connected with the input of one of the five counters. All counters have one frequency which is the same frequency " $f$ ' for incrementing their values. The TD output is also connected with the control logic module (CLM). The counters have two control inputs: one input for the signals emerged from the output of the TD, and the other one, called START, for the signals coming from the CLM as shown in the schematic diagram of OCM as seen in Fig.5.

When the output signal of a certain TD is at level $\mathrm{H}$, and the signal at START is also at $\mathrm{H}$, then the corresponding counter increments its value. The increment proceeds until either one of the two control input levels is switched to $L$. Therefore, the final value of the counter is proportional to the period of time during which the signals at the two control inputs are simultaneously indicating the level $\mathrm{H}$. The final value of the counter is read and then cleared.

The algorithm that controls the process of determining operates in a cycles countering. Each cycle is initiated by the transition $\mathrm{L} \rightarrow \mathrm{H}$ of the signal level at START which occurs at the instant where the photodiode $\mathrm{C}$ has just slipped out of a bright speckle. At that time two others photodiodes have already located over the same speckle indicating $\mathrm{H}$ level at their TD outruitp.

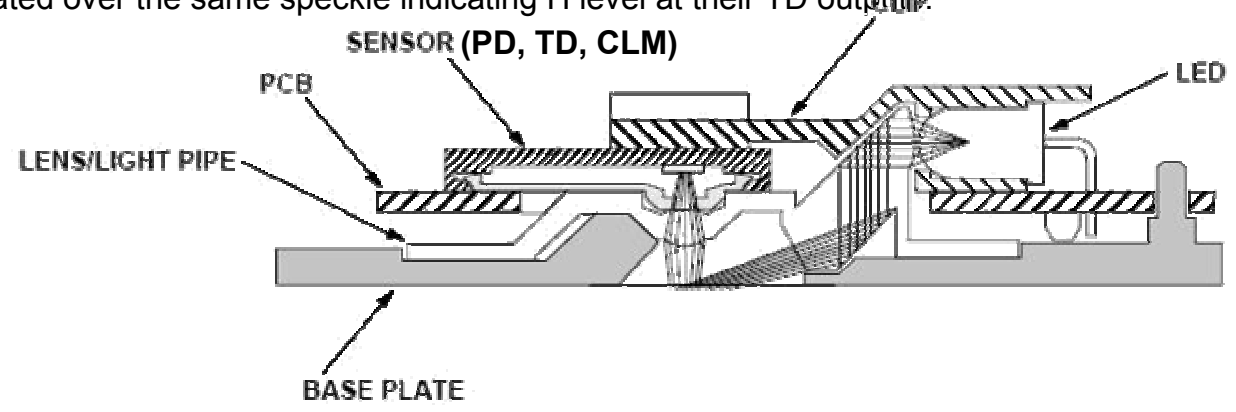

Fig. 5. Schematic Diagram of Optical Computer Mouse (OCM)

This configuration becomes possible after accurate regulation of the optical sensor according to the relation $a \leq \delta$. It is now the turn of the CLM to verify its input levels in order to find out which of these PDs are working in good situation. This knowledge is determinant of the quadrant where the motion of the surface is directed. The next step of the CLM is to enable the increment of the respective counters. 
Each counter increment lasts until the corresponding PD comes to the end of one speckle, which causes the transition $\mathrm{H} \rightarrow \mathrm{L}$ at the output of the associated TD. After the termination of both increments the transition $\mathrm{H} \rightarrow \mathrm{L}$ of the signal at START ends the cycle. The two final readings of the counters are stored. The ratio between the two readings is used to determine the direction of $(v)$. In order to achieve that we will consider the situation where a large number of speckles will detected when the device moves across the whole speckle pattern. The collected data are used to compute the average value of $\delta$.This means that we will not apply the calculations to one speckle grain, we will use the calculations for a large number of speckles considering that the standard deviation of the errors will be zero i.e. the errors will cancel each others.

The following table (1) presents the values of the normalized or relative response of the optical mouse sensor for different wavelengths of used LEDs, and the wavelength responsivity is shown in Fig. 6 .

Table (1):

\begin{tabular}{|c|c|c|c|c|c|c|c|}
\hline Wavelength $\lambda(\mathrm{nm})$ & 400 & 500 & 600 & 700 & 800 & 900 & 1000 \\
\hline Relative response & 0.344 & 0.545 & 0.713 & 0.892 & 1.011 & 0.773 & 0.352 \\
\hline
\end{tabular}

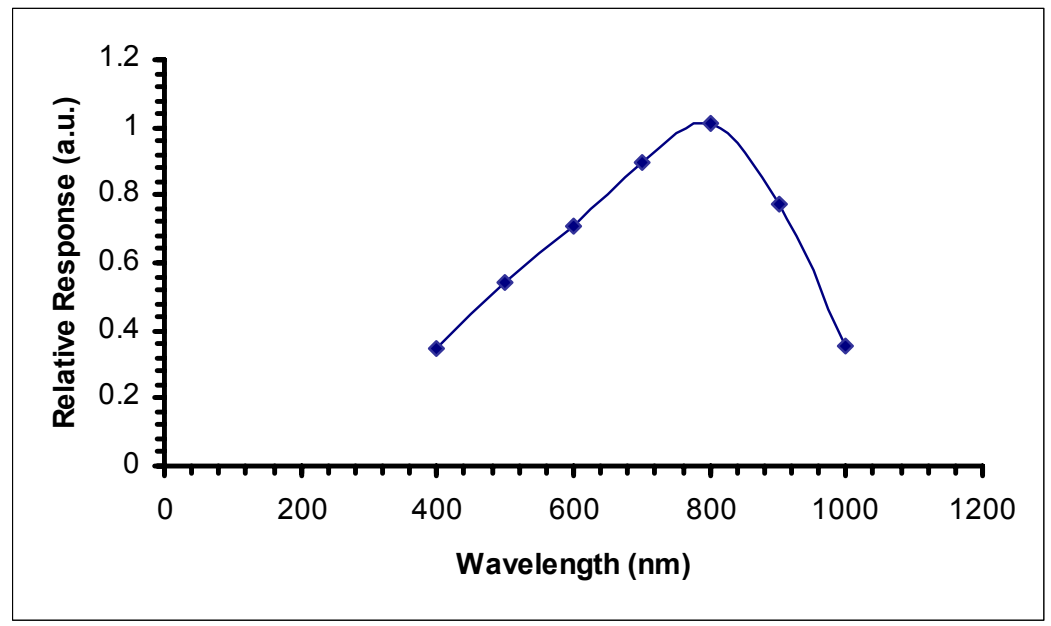

Fig 6. Wavelengths responsivity of OCM sensor

\section{Experimental Studies and results:}

This work describes the light speckles produced from different colour sources (LEDs and lasers) with different glass diffusers (GT1, GT3, GT4, GT5, GT6 and thin holographic plate) as the different types of pad surfaces to be able to design and implement a new optical computer mouse (OCM).

In the experimental set-up, we replace the standard optical mouse light sources with our different type of light sources [He-Ne laser, Diode laser and (Red, Blue, Green, Yellow and Orange LEDs)] to illuminate the different glass diffusers mentioned above, and by using a high performance colour coupled charge device (CCD camera, Model 8313-1000, COHU Company) the images of laser speckle patterns are captured as shown Fig $7(a, b, c, d, e, f)$.

\section{For GT1 diffuser:}






He-Ne laser

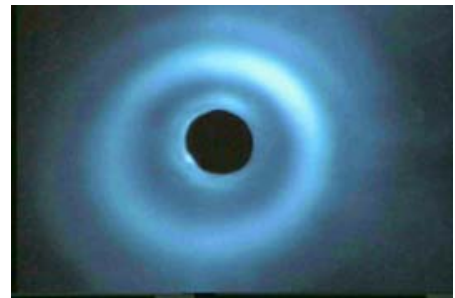

Blue LED

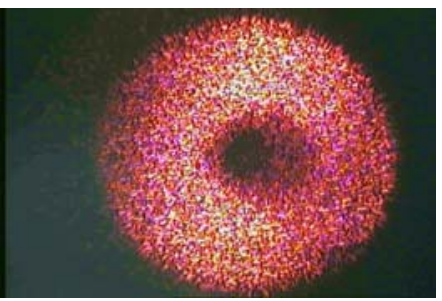

Diode laser

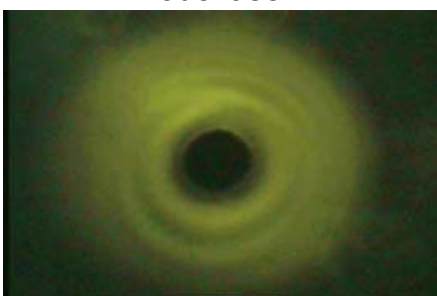

Green LED

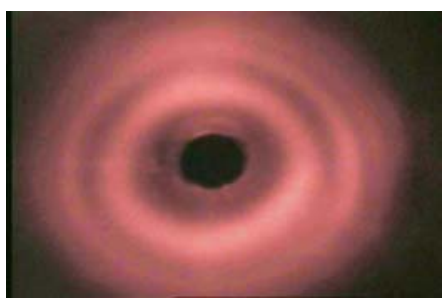

Red LED



Yellow LED

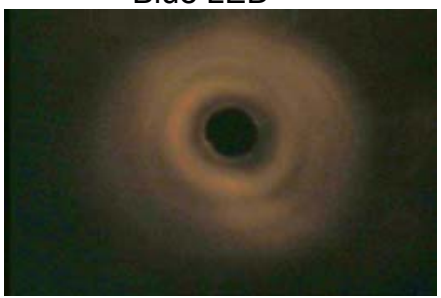

Orange LED

Fig. 7(a) The images of laser speckle patterns obtained by using GT1 diffuser.

\section{For GT3 diffuser:}

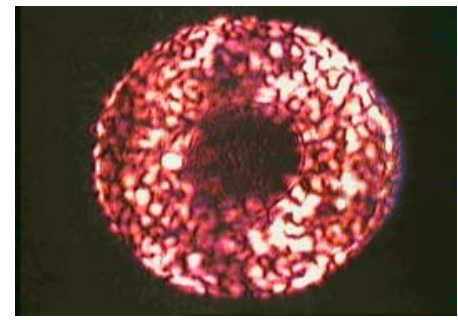

$\mathrm{He}-\mathrm{Ne}$ laser

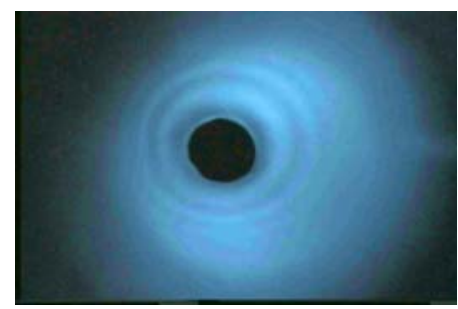

Blue LED

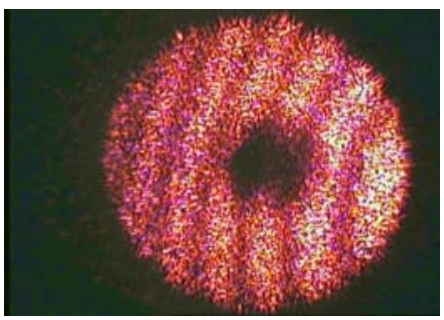

Diode laser

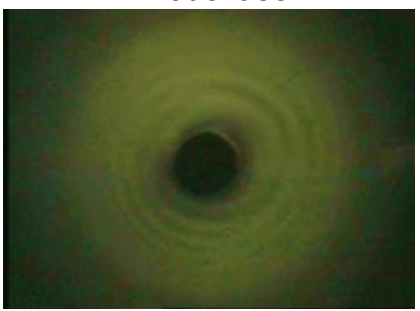

Green LED

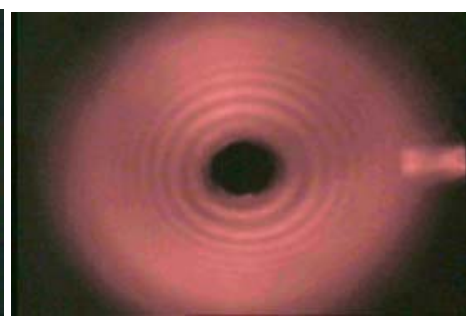

Red LED

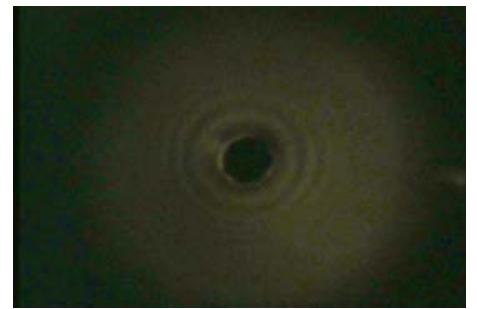

Yellow LED

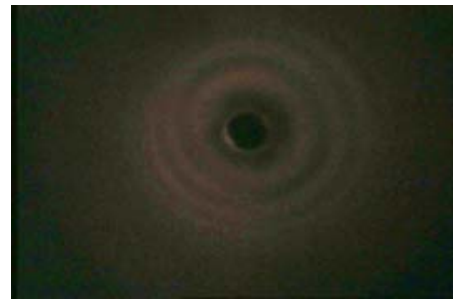

Orange LED

Fig. 7(b) The images of laser speckle patterns obtained by using GT3 diffuser.

\section{For GT4 diffuser:}




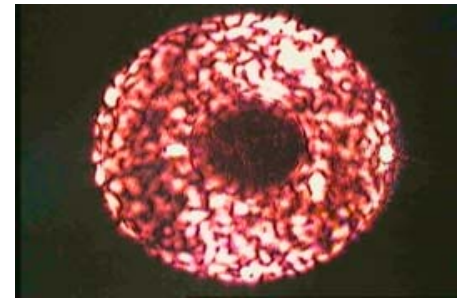

$\mathrm{He}-\mathrm{Ne}$ laser

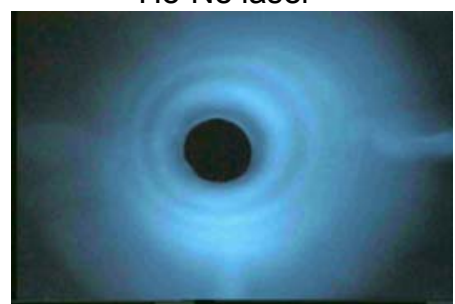

Blue LED

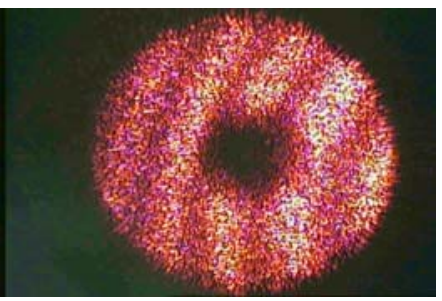

Diode laser

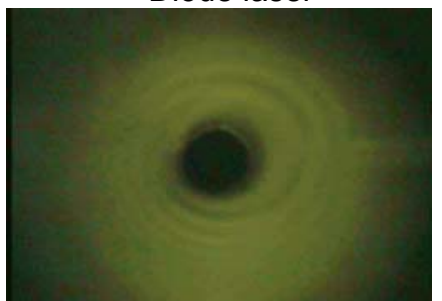

Green LED

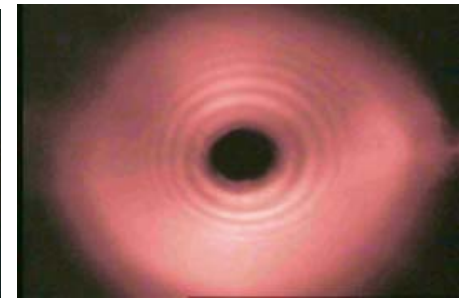

Red LED

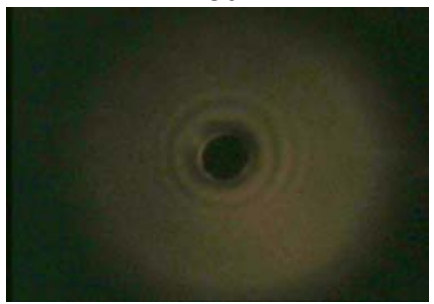

Yellow LED

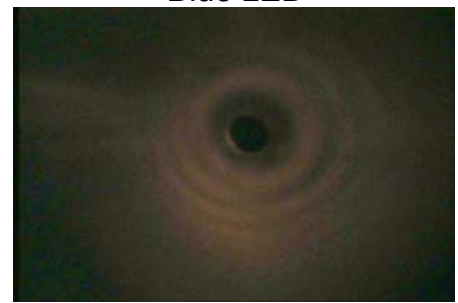

Orange LED

Fig. 7(c) The images of laser speckle patterns obtained by using GT4 diffuser.

\section{For GT5 diffuser:}



$\mathrm{He}-\mathrm{Ne}$ laser

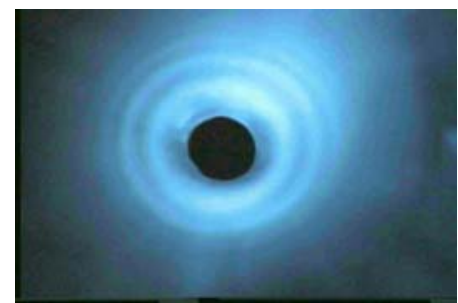

Blue LED

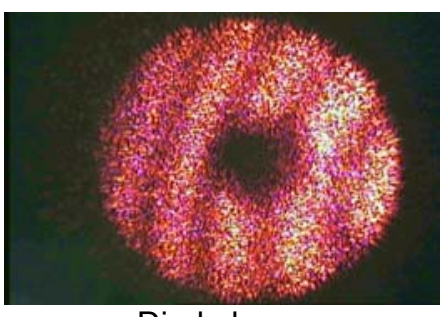

Diode laser

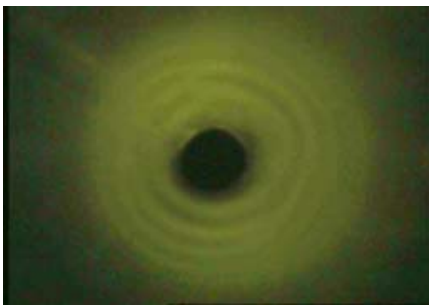

Green LED



Red LED

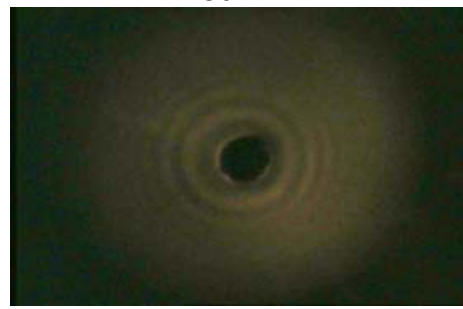

Yellow LED

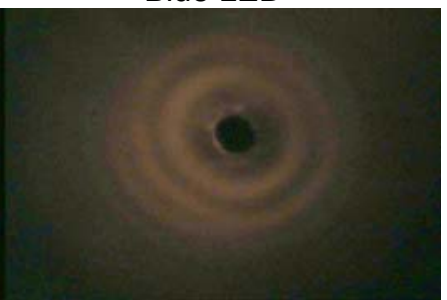

Orange LED

Fig. 7(d) The images of laser speckle patterns obtained by using GT5 diffuser. For GT6 diffuser: 


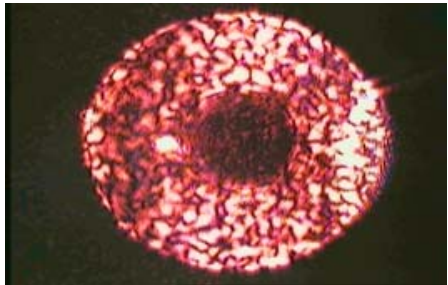

He-Ne laser

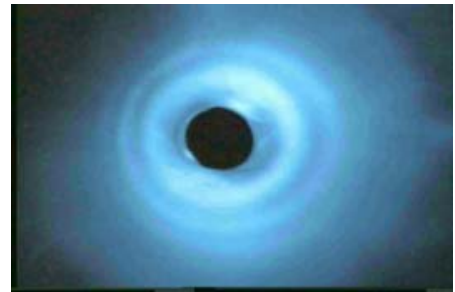

Blue LED

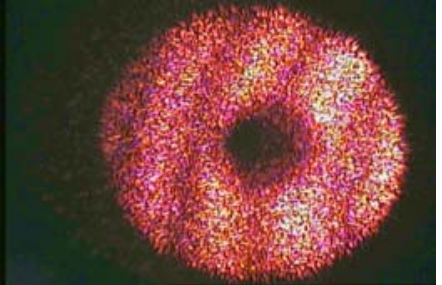

Diode laser

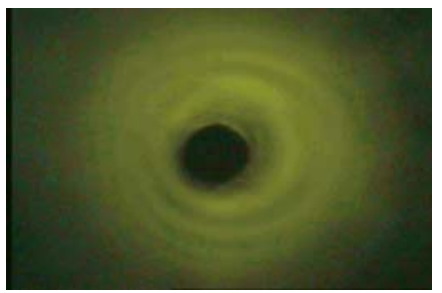

Green LED

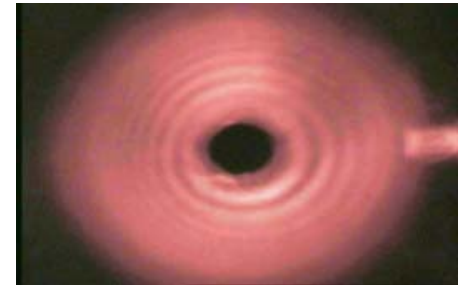

Red LED

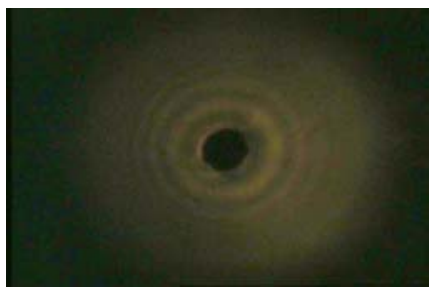

Yellow LED

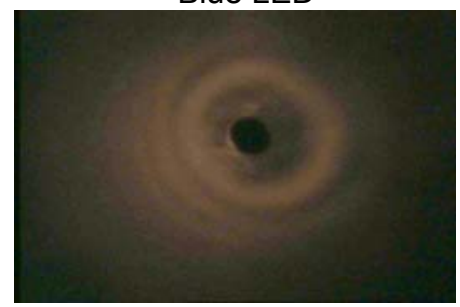

Orange LED

Fig. 7(e) The images of laser speckle patterns obtained by using GT6 diffuser.

\section{For thin film diffuser:}

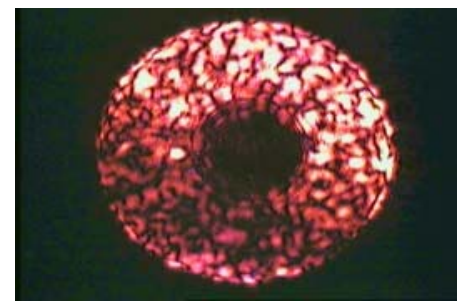

He-Ne laser

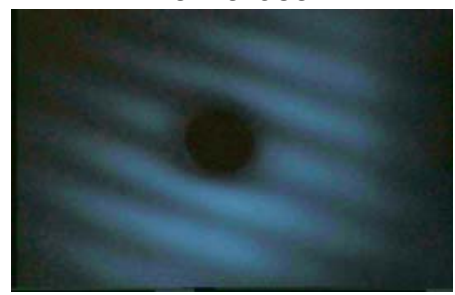

Blue LED

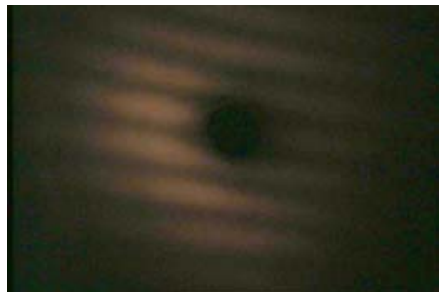

Orange LED

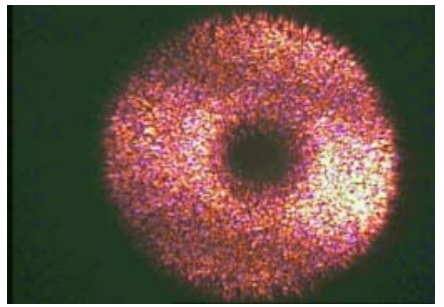

Diode laser

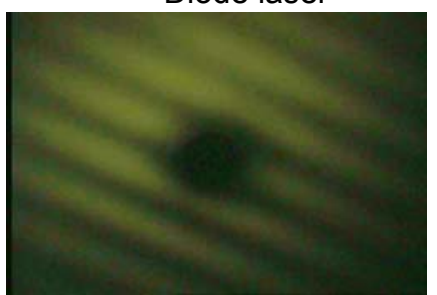

Green LED

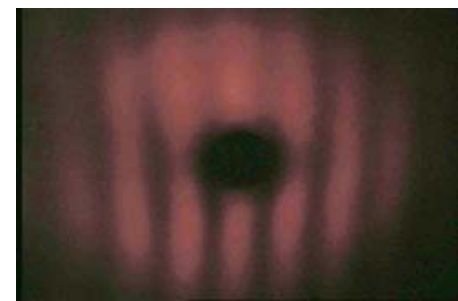

Red LED



Yellow LED

Fig. 7(f) The images of laser speckle patterns obtained by using thin holographic plate.

\section{Discussion and conclusions:}


In this work the images of a speckle patterns produced by illuminating the different types of pad surfaces with different types of light sources (LEDs and lasers) have been investigated experimentally and theoretically. As seen in Fig.7, the images of the speckle patterns produced by using He-Ne laser ( $\lambda=632.8 \mathrm{~nm})$ and diode laser $(\lambda=570 \mathrm{~nm})$ appears resolved a bright and dark spots (referred as laser speckles) this is due to the narrow band wavelength spectrum (highly monochromatic). For the case where the images of the speckle patterns produced by using different color LEDs, due to the wide band wavelength spectrum (poorly monochromatic) the bright and dark spots superimposed and cancelled with each other. This leads to the formation of interference fringes pattern resulting from double exposure Young's interference.

Computer image processing has been introduced to measure and analyze the laser speckle size by using MatLab software. In MatLab program, we select the suitable gray scale by choosing the best redgreen-blue (RGB) factors according to the wavelength value for each type of light sources, i.e., for HeNe laser with $\lambda=632.8 \mathrm{~nm}$ has $R=0.8681, G=0.1041, B=0.0278$ and for Diode laser with $\lambda=570 \mathrm{~nm}$ has $R=0.7486, G=0.1938, B=0.0576$ ) as given from the International Telecommunication Organization (ITU-R709 for CRT phosphorus, Tirinton-SONY Model), then by selecting a suitable intensity threshold level we convert this digital image $(0-255)$ to a binary image ( 0 and 1$)$, where zeros are for the intensity values less than the threshold value (150) and ones for the remaining values.

By comparing the number of zeros (dark spots) and ones (bright spots) in the obtained binary image, we are able to determine the responsivity of OCM sensor for certain type of light source. So, from these data we can choose the best light source for a certain pad surface.

From the experimental results we conclude that the best laser speckle intensity are obtained by using laser sources (He-Ne laser and Diode laser), but these sources are not commercial and relatively expensive more than other type of light sources like LEDs. Also from the obtained different speckle images for different types of similar output power LEDs, we introduce that the intensity distribution of the speckle pattern at a specified distance for pad surfaces:

(i) [GT1, GT3, GT4] is high for [red and blue LEDs], moderate for [green and orange LEDs] and low for [yellow LED].

(ii) [GT5], it is high for [red LED], moderate for [blue, green and orange LEDs] and low for [yellow LED].

(iii) [GT6], it is high for [blue LED], moderate for [red, green and orange LEDs] and low for [yellow LED].

(iv) [Thin holographic plate], it is high for [green LED], moderate for [red, blue and orange LEDs] and low for [yellow LED]. 


\section{References:}

1) Barakat, N; Merzkirch, Wernikinck, W, W.; El-Ghandoor, H Optik 1986, vol, 74-3.

2) Barakat, N, N; Hamed, A.M.; El-Ghandoor, H Optik 1987, vol, 76-3.

3) El-Ghandoor, H Optik 1987, vol, 76-3.

4) Li, E.L; Tieu, A.K.; Wang, K.F. Optics Communications 2003, vol, 219.

5) El-Ghandoor, H.; Hamed, A.M International Symposium on the Technologies for Optoelectronics, November 1987, Cannes, France,

6) M. Francon, "Laser Speckle and Application in Optics" Academic Press, NY, 1979. 